\title{
THE FIRST SZEGŐ LIMIT THEOREM FOR NON-SELFADJOINT OPERATORS IN THE FØLNER ALGEBRA
}

\author{
ALBRECHT BÖTTCHER and PETER OTTE
}

\begin{abstract}
We determine the first order asymptotics of the trace of $f\left(P_{n} U P_{n}\right)$ and the determinant det $P_{n} U P_{n}$ for operators $U$ belonging to the Følner algebra associated with the sequence $\left\{P_{n}\right\}$ and satisfying an "index zero" condition. We present three different proofs of the main result in the case where $U$ is a normal operator.
\end{abstract}

\section{Introduction}

Let $H$ be a separable Hilbert space. We denote by $\|\cdot\|,\|\cdot\|_{1},\|\cdot\|_{2}$ the operator norm, the trace norm, and the Hilbert-Schmidt norm, respectively. We fix a sequence $\left\{P_{n}\right\}$ of orthogonal projections on $H$ such that $R_{n}:=\operatorname{dim} \operatorname{ran} P_{n}<$ $\infty$ for all $n$ and we put $Q_{n}=I-P_{n}$. For $B \in \mathscr{B}(H)$,

$$
\begin{aligned}
& \left\|P_{n} B Q_{n}\right\|_{2}^{2}=\operatorname{tr}\left(Q_{n} B^{*} P_{n} B Q_{n}\right)=\operatorname{tr}\left(P_{n} B Q_{n} B^{*} P_{n}\right), \\
& \left\|Q_{n} B P_{n}\right\|_{2}^{2}=\operatorname{tr}\left(P_{n} B^{*} Q_{n} B P_{n}\right) .
\end{aligned}
$$

The Følner algebra $\mathscr{F}\left(\left\{P_{n}\right\}\right)$ associated with $\left\{P_{n}\right\}$ is the set of all operators $B$ in $\mathscr{B}(H)$ for which

$$
\lim _{n \rightarrow \infty} \frac{\left\|P_{n} B Q_{n}\right\|_{2}^{2}}{R_{n}}=0, \quad \lim _{n \rightarrow \infty} \frac{\left\|Q_{n} B P_{n}\right\|_{2}^{2}}{R_{n}}=0 .
$$

The set $\mathscr{F}\left(\left\{P_{n}\right\}\right)$ is a unital $C^{*}$-subalgebra of $\mathscr{B}(H)$. For $B \in \mathscr{B}(H)$, we consider the sequence $\left\{P_{n} B P_{n}\right\}$. We are interested in a first order asymptotics of the trace $\operatorname{tr} f\left(P_{n} B P_{n}\right)$ for appropriate functions $f$ and in particular in the case $f(\lambda)=\log \lambda$, which amounts to considering the $\operatorname{determinant} \operatorname{det}\left(P_{n} B P_{n}\right)$.

Two standard situations are $H=\ell^{2}(\mathrm{Z})$ and

(1) $P_{n}:\left\{x_{j}\right\}_{j=-\infty}^{\infty} \mapsto\left\{\ldots, 0, x_{-n}, \ldots, x_{0}, \ldots, x_{n}, 0, \ldots\right\} \quad\left(R_{n}=2 n+1\right)$,
(2) $P_{n}:\left\{x_{j}\right\}_{j=-\infty}^{\infty} \mapsto\left\{\ldots, 0, x_{0}, \ldots, x_{n-1}, 0, \ldots\right\} \quad\left(R_{n}=n\right)$.

Received July 27, 2004. 
In these two cases, $\mathscr{F}\left(\left\{P_{n}\right\}\right)$ contains all banded operators and all Laurent operators. A banded operator is an operator that is induced by a banded matrix. A Laurent operator $L(\varphi)$ is given by a matrix of the form $\left(\varphi_{j-k}\right)_{j, k=-\infty}^{\infty}$ where the $\varphi_{k}$ 's are the Fourier coefficients of a bounded function $\varphi$, that is,

$$
\varphi_{k}=\frac{1}{2 \pi} \int_{0}^{2 \pi} \varphi\left(e^{i \theta}\right) e^{-i k \theta} d \theta \quad(k \in \mathbf{Z})
$$

with $\varphi$ in $L^{\infty}$ on the complex unit circle T. Notice that $L(\varphi)$ is unitarily equivalent in an obvious way to the operator of multiplication by $\varphi$ on $L^{2}(\mathrm{~T})$. If $P_{n}$ is as in (2), then the operators $P_{n} L(\varphi) P_{n} \mid$ ran $P_{n}$ may be identified with $n \times n$ Toeplitz matrices $T_{n}(\varphi):=\left(\varphi_{j-k}\right)_{j, k=0}^{n-1}$. The classical first Szegó limit theorem [18] states that if $\varphi$ is real-valued and essinf $\varphi>0$ on T, then

$$
\log \operatorname{det} T_{n}(\varphi)=n(\log \varphi)_{0}+o(n) \quad \text { as } \quad n \rightarrow \infty,
$$

where $(\log \varphi)_{0}$ is the 0 th Fourier coefficient of $\log \varphi$. (Notice that $\operatorname{det} T_{n}(\varphi)$ is positive for $\varphi>0$, so that the logarithm is well-defined.) Extensions of (3) to more general situations, mainly concerning either Toeplitz-like or selfadjoint operators $B$ in place of $L(\varphi)$ have been studied by many authors. References [1], [2], [4]-[17], and [19]-[21] are a few exemplary works of the business.

We here prove an analogue of (3) for operators in $\mathscr{F}\left(\left\{P_{n}\right\}\right)$ that are a power of an operator whose numerical range is separated away from zero and that are subject to an additional stability requirement. Suppose, for example, the operator under consideration is the Laurent operator $L(\varphi)$ with a continuous function $\varphi: \mathrm{T} \rightarrow \mathrm{C}$. The operator $L(\varphi)$ is the power of an operator $L(\psi)$ with a continuous function $\psi$ for which the convex hull of $\psi(\mathrm{T})$ does not contain the origin if and only if the winding number (= index) of $\varphi$ about the origin is zero. Consequently, the assumption of our main theorem (Theorem 3.2) may be interpreted as an "index zero" condition. Notice also that the operator $L(\varphi)$ is selfadjoint if and only if $\varphi$ is real-valued. But this operator is always normal. Thus, when dealing with the problem considered here, passage from selfadjoint to normal operators is in fact quite a nontrivial step, because it includes passing from Hermitian Toeplitz matrices to arbitrary Toeplitz matrices as a special case.

\section{A general trace formula}

Let $B$ be an operator in $\mathscr{B}(H)$. The sequence $\left\{P_{n} B P_{n}\right\}$ is said to be stable if the operators $P_{n} B P_{n} \mid$ ran $P_{n}$ are invertible for all sufficiently large $n$, say $n \geq n_{0}$, and $\sup _{n \geq n_{0}}\left\|\left(P_{n} B P_{n}\right)^{-1} P_{n}\right\|<\infty$.

The spectrum of an operator $B \in \mathscr{B}(H)$ will be denoted by $\sigma(B)$. We write $\lambda-B$ and $\lambda-P_{n} B P_{n}$ for $\lambda I-B$ and $\left(\lambda I-P_{n} B P_{n}\right) \mid$ ran $P_{n}$, respectively. 
THEOREM 2.1. Let $K$ be a compact subset of $\mathrm{C}$ and let $\Omega \subset \mathrm{C}$ be a bounded open set with a smooth boundary $\partial \Omega$ that contains $K$. Let $f$ be analytic in $\Omega$ and continuous on the closure of $\Omega$. Let finally $U \in \mathscr{F}\left(\left\{P_{n}\right\}\right)$ and suppose $\sigma(U) \subset K$ and $\left\{P_{n}(\lambda-U) P_{n}\right\}$ is stable for all $\lambda \in \partial \Omega$. Then

$$
\operatorname{tr} f\left(P_{n} U P_{n}\right)=\operatorname{tr} P_{n} f(U) P_{n}+o\left(R_{n}\right) \quad \text { as } n \rightarrow \infty .
$$

The proof is based on three lemmas. The first lemma is the basic trick of our approach. The other two lemmas are needed to make some estimates in the proof of Theorem 2.1 uniform.

Lemma 2.2. Let $B \in \mathscr{B}(H)$ be invertible and let $P$ and $Q$ be complementary projections on $H$. Then $P B P \mid \operatorname{ran} P$ is invertible if and only if $Q B^{-1} Q \mid \operatorname{ran} Q$ is invertible. In that case

$$
\begin{aligned}
(P B P)^{-1} P & =P B^{-1} P-P B^{-1} Q\left(Q B^{-1} Q\right)^{-1} Q B^{-1} P, \\
\left(Q B^{-1} Q\right)^{-1} Q & =Q B Q-Q B P(P B P)^{-1} P B Q .
\end{aligned}
$$

Proof. See [5, Proposition 7.15] or [6, Lemma 2.9], for example.

Lemma 2.3. Let $B \in \mathscr{B}(H), \lambda \in \mathrm{C}$, and suppose $\left\{P_{n}(\lambda-B) P_{n}\right\}$ is stable. Then there exist $n_{0} \in \mathrm{N}, M<\infty, \varepsilon>0$ such that

$$
\left\|\left(P_{n}(\mu-B) P_{n}\right)^{-1} P_{n}\right\| \leq M
$$

for $n \geq n_{0}$ and $|\mu-\lambda|<\varepsilon$.

Proof. Put $B_{n}=P_{n} B P_{n} \mid \operatorname{ran} P_{n}$. Suppose that $\lambda-B_{n}$ is invertible and that $\left\|\left(\lambda-B_{n}\right)^{-1} P_{n}\right\| \leq N<\infty$ for all $n \geq n_{0}$. With $\mu=\lambda+\delta$,

$$
\mu-B_{n}=\lambda+\delta-B_{n}=\left(\lambda-B_{n}\right)\left(I+\delta\left(\lambda-B_{n}\right)^{-1}\right),
$$

and hence $\mu-B_{n}$ is invertible for all $n \geq n_{0}$ whenever $|\delta| N<1$. For these $\delta$ we get

$$
\left\|\left(\mu-B_{n}\right)^{-1} P_{n}\right\| \leq \sum_{k=0}^{\infty}|\delta|^{k}\left\|\left(\lambda-B_{n}\right)^{-1}\right\|^{k+1} \leq \frac{N}{1-|\delta| N},
$$

which yields the assertion with $\varepsilon=1 /(2 N)$ and $M=2 N$.

Lemma 2.4. Let $B \in \mathscr{B}(H), \lambda \in \mathrm{C} \backslash \sigma(B)$, and suppose

$$
\lim _{n \rightarrow \infty} \frac{\left\|P_{n}(\lambda-B)^{-1} Q_{n}\right\|_{2}^{2}}{R_{n}}=0 .
$$


Then there exists an $\varepsilon>0$ such that

$$
\lim _{n \rightarrow \infty} \frac{\left\|P_{n}(\mu-B)^{-1} Q_{n}\right\|_{2}^{2}}{R_{n}}=0
$$

uniformly for $|\mu-\lambda|<\varepsilon$.

Proof. Let again $\mu=\lambda+\delta$ and suppose $|\delta|\left\|(\lambda-B)^{-1}\right\|<1$. Then

$$
\begin{aligned}
\left\|P_{n}(\mu-B)^{-1} Q_{n}\right\|_{2} & =\left\|P_{n}(\lambda+\delta-B)^{-1} Q_{n}\right\|_{2} \\
& =\left\|P_{n} \sum_{k=0}^{\infty}(-1)^{k} \delta^{k}\left[(\lambda-B)^{-1}\right]^{k+1} Q_{n}\right\|_{2} \\
& \leq \sum_{k=0}^{\infty}|\delta|^{k}\left\|P_{n}\left[(\lambda-B)^{-1}\right]^{k+1} Q_{n}\right\|_{2} \\
& \leq \sum_{k=0}^{\infty}|\delta|^{k}(k+1)\left\|(\lambda-B)^{-1}\right\|^{k}\left\|P_{n}(\lambda-B)^{-1} Q_{n}\right\|_{2} \\
& =\frac{\left\|P_{n}(\lambda-B)^{-1} Q_{n}\right\|_{2}}{\left(1-|\delta|\left\|(\lambda-B)^{-1}\right\|\right)^{2}},
\end{aligned}
$$

which gives the assertion with $\varepsilon=1 /\left(2\left\|(\lambda-B)^{-1}\right\|\right)$.

Proof of Theorem 2.1. We have

(6) $\operatorname{tr} f\left(P_{n} U P_{n}\right)-\operatorname{tr} P_{n} f(U) P_{n}$

$$
=\frac{1}{2 \pi i} \int_{\partial \Omega} f(\lambda) \operatorname{tr}\left[\left(P_{n}(\lambda-U) P_{n}\right)^{-1} P_{n}-P_{n}(\lambda-U)^{-1} P_{n}\right] d \lambda .
$$

By (4), the absolute value of (6) does not exceed

$\frac{1}{2 \pi} \int_{\partial \Omega}|f(\lambda)|\left|\operatorname{tr}\left[P_{n}(\lambda-U)^{-1}\left(Q_{n}(\lambda-U)^{-1} Q_{n}\right)^{-1} Q_{n}(\lambda-U)^{-1} P_{n}\right]\right||d \lambda|$, and since $|\operatorname{tr}(A B C)| \leq\|A\|_{2}\|B\|\|C\|_{2}$, this is at most

$$
\begin{aligned}
\frac{1}{2 \pi} \int_{\partial \Omega}|f(\lambda)|\left\|\left(Q_{n}(\lambda-U)^{-1} Q_{n}\right)^{-1} Q_{n}\right\| & \\
& \times\left\|P_{n}(\lambda-U)^{-1} Q_{n}\right\|_{2}\left\|Q_{n}(\lambda-U)^{-1} P_{n}\right\|_{2}|d \lambda| .
\end{aligned}
$$

The sequence $\left\{P_{n}(\lambda-U) P_{n}\right\}$ is stable for each $\lambda \in \partial \Omega$. Since $\partial \Omega$ is compact, Lemma 2.3 implies that there are $n_{0} \in \mathrm{N}$ and $M<\infty$ such that

$$
\left\|\left(P_{n}(\lambda-U) P_{n}\right)^{-1} P_{n}\right\| \leq M
$$


for all $n \geq n_{0}$ and all $\lambda \in \partial \Omega$. Identity (5) therefore implies that

$$
\left\|\left(Q_{n}(\lambda-U)^{-1} Q_{n}\right)^{-1} Q_{n}\right\| \leq\|\lambda-U\|+\|\lambda-U\|^{2} M \leq N<\infty
$$

for all $n \geq n_{0}$ and all $\lambda \in \partial \Omega$. Since $U \in \mathscr{F}\left(\left\{P_{n}\right\}\right)$ and $\lambda-U$ is invertible, the inverse $(\lambda-U)^{-1}$ belongs to the $C^{*}$-algebra $\mathscr{F}\left(\left\{P_{n}\right\}\right)$ for each $\lambda \in \partial \Omega$. Lemma 2.4 and the compactness of $\partial \Omega$ therefore yield that

$$
\max _{\lambda \in \partial \Omega} \frac{\left\|P_{n}(\lambda-U)^{-1} Q_{n}\right\|_{2}}{\sqrt{R_{n}}} \frac{\left\|Q_{n}(\lambda-U)^{-1} P_{n}\right\|_{2}}{\sqrt{R_{n}}} \rightarrow 0
$$

as $n \rightarrow \infty$. This gives the assertion.

\section{Operators with good numerical range}

Here is a first consequence of Theorem 2.1.

Corollary 3.1. Let $U \in \mathscr{F}\left(\left\{P_{n}\right\}\right)$ and suppose the closure of the numerical range $\mathscr{H}(U):=\{(U x, x):\|x\|=1\}$ does not contain the origin. Then $U=e^{A}$ for some $A \in \mathscr{F}\left(\left\{P_{n}\right\}\right)$ and

$$
\log \operatorname{det} P_{n} U P_{n}=\operatorname{tr} P_{n} A P_{n}+o\left(R_{n}\right) \quad \text { as } n \rightarrow \infty,
$$

where $\log$ is any branch of the logarithm that is analytic on $\operatorname{clos} \mathscr{H}(U)$.

Proof. We employ Theorem 2.1 with $K=\operatorname{clos} \mathscr{H}(U)$. We may without loss of generality assume that $K$ is a subset of the right open half-plane. The spectrum of $U$ is contained in $K$. Let $\Omega \supset K$ be a bounded open subset of the right open half-plane with a smooth boundary $\partial \Omega$. The function $f(\lambda)=\log \lambda$ is analytic in $\Omega$ and continuous on the closure of $\Omega$. Thus, $U=e^{A}$ with

$$
A=\frac{1}{2 \pi i} \int_{\partial \Omega}(\log \lambda)(\lambda-U)^{-1} d \lambda .
$$

If $\lambda \in \partial \Omega$, then $0 \notin \lambda-\operatorname{clos} \mathscr{H}(U)=\operatorname{clos} \mathscr{H}(\lambda-U)$. This implies that $\lambda-U=\alpha(I+S)$ with $\alpha \in \mathrm{C} \backslash\{0\}$ and $\|S\|<1$ and hence

$$
\left\|\left(P_{n}(\lambda-U) P_{n}\right)^{-1} P_{n}\right\| \leq \frac{1}{|\alpha|} \sum_{k=0}^{\infty}\left\|P_{n} S P_{n}\right\|^{k} \leq \frac{1}{|\alpha|(1-\|S\|)},
$$

which shows that $\left\{P_{n}(\lambda-U) P_{n}\right\}$ is stable for every $\lambda \in \partial \Omega$ (this argument is from [10, Section II.5]). The corollary is now immediate from Theorem 2.1 and the identity $\log \operatorname{det} P_{n} U P_{n}=\operatorname{tr} \log P_{n} U P_{n}$.

The following result concerns powers of operators with good numerical range. The stability requirement in that result is nasty at the first glance, but in 
the next section we will see that the result is not true without this additional condition.

THEOREM 3.2. Let $U \in \mathscr{F}\left(\left\{P_{n}\right\}\right)$ and suppose the origin does not belong to the closure of $\mathscr{H}(U)$. If $k \in \mathrm{N}$ and $\left\{P_{n} U^{k} P_{n}\right\}$ is stable, then

$$
\log \left|\operatorname{det} P_{n} U^{k} P_{n}\right|=k \log \left|\operatorname{det} P_{n} U P_{n}\right|+o\left(R_{n}\right) \quad \text { as } n \rightarrow \infty \text {. }
$$

Proof. It is easy to show that

$$
\left\|P_{n} B^{k} P_{n}-\left(P_{n} B P_{n}\right)^{k}\right\|_{1} \leq k\|B\|^{k}\left\|P_{n} B Q_{n}\right\|_{1}
$$

for every $B \in \mathscr{B}(H)$. Put $L_{n}:=P_{n} U^{k} P_{n}-\left(P_{n} U P_{n}\right)^{k}$. Using (9) and taking into account that $\left\|P_{n} B\right\|_{1} \leq\left\|P_{n}\right\|_{2}\left\|P_{n} B\right\|_{2}=\sqrt{R_{n}}\left\|P_{n} B\right\|_{2}$, we get

$$
\begin{aligned}
\frac{\left\|L_{n}\right\|_{1}}{R_{n}} & \leq k\|U\|^{k} \frac{\left\|P_{n}\right\|_{2}\left\|P_{n} U Q_{n}\right\|_{2}}{R_{n}} \\
& =k\|U\|^{k} \frac{\left\|P_{n} U Q_{n}\right\|_{2}}{\sqrt{R_{n}}} \\
& =o(1) .
\end{aligned}
$$

Furthermore,

$$
\operatorname{det} P_{n} U^{k} P_{n}=\left(\operatorname{det} P_{n} U P_{n}\right)^{k} \operatorname{det}\left(I+\left(P_{n} U P_{n}\right)^{-k} L_{n}\right),
$$

and since the closure of $\mathscr{H}(U)$ does not contain the origin, we may conclude as in the proof of Corollary 3.1 that the sequence $\left\{P_{n} U P_{n}\right\}$ is stable. Consequently,

$$
\left|\operatorname{det}\left(I+\left(P_{n} U P_{n}\right)^{-k} L_{n}\right)\right| \leq e^{\left\|\left(P_{n} U P_{n}\right)^{-k} L_{n}\right\|_{1}} \leq e^{M\left\|L_{n}\right\|_{1}}
$$

with some constant $M<\infty$. It follows that

$$
\limsup _{n \rightarrow \infty} \frac{1}{R_{n}}\left(\log \left|\operatorname{det} P_{n} U^{k} P_{n}\right|-k \log \left|\operatorname{det} P_{n} U P_{n}\right|\right) \leq 0 .
$$

On the other hand,

$$
\left(\operatorname{det} P_{n} U P_{n}\right)^{k}=\left(\operatorname{det} P_{n} U^{k} P_{n}\right) \operatorname{det}\left(I-\left(P_{n} U^{k} P_{n}\right)^{-1} L_{n}\right) .
$$

Since $\left\{P_{n} U^{k} P_{n}\right\}$ is stable by assumption, the same argument as above yields that

$$
\limsup _{n \rightarrow \infty} \frac{1}{R_{n}}\left(k \log \left|\operatorname{det} P_{n} U P_{n}\right|-\log \left|\operatorname{det} P_{n} U^{k} P_{n}\right|\right) \leq 0 .
$$

Combining (10) and (11) we arrive at the assertion. 
The following corollary is well known (see, e.g., [5], [10], [12]). We cite it in order to illustrate Theorem 3.2 by a concrete realization.

Corollary 3.3. Let $\varphi \in L^{\infty}(\mathrm{T})$ and suppose $\left\{T_{n}(\varphi)\right\}$ is stable. Then

$$
\log \left|\operatorname{det} T_{n}(\varphi)\right|=n(\log |\varphi|)_{0}+o(n) \quad \text { as } n \rightarrow \infty .
$$

Proof. The stability of $\left\{T_{n}(\varphi)\right\}$ implies that essinf $|\varphi|>0$ on T. Write $\varphi=$ $|\varphi| e^{i b}$ with a real-valued function $b: \mathrm{T} \rightarrow(-\pi, \pi]$ and put $\psi=|\varphi|^{1 / 3} e^{i b / 3}$. The operator $U=L(\psi)$ is normal. The closure of $\mathscr{H}(U)$ is therefore the convex hull of the spectrum. As the spectrum of $L(\psi)$ is the essential range of $\psi$, we conclude that clos $\mathscr{H}(U)$ is a subset of the right open half-plane. From Corollary 3.1 we deduce that

$$
\log \left|\operatorname{det} P_{n} L(\psi) P_{n}\right|=\frac{1}{3} \operatorname{tr} P_{n} L(\log |\varphi|) P_{n}+o(n)
$$

and Theorem 3.2 shows that

$$
\log \left|\operatorname{det} P_{n} L(\varphi) P_{n}\right|=3 \log \left|\operatorname{det} P_{n} L(\psi) P_{n}\right|+o(n) .
$$

The last two relations clearly imply the assertion.

Note that if $\varphi$ is real-valued and essinf $\varphi>0$ on $\mathrm{T}$, then $\left\{T_{n}(\varphi)\right\}$ is stable and det $T_{n}(\varphi)>0$. Hence Corollary 3.3 contains the first Szegó limit theorem as a special case.

For a piecewise continuous function $\varphi \in L^{\infty}(\mathrm{T})$, let $\varphi^{\#}(\mathrm{~T})$ be the naturally oriented curve that consists of the components of $\varphi(\mathrm{T})$ connected by straight segments at jumps. The sequence $\left\{T_{n}(\varphi)\right\}$ is known to be stable if and only if $\varphi^{\#}(\mathrm{~T})$ does not contain the origin and has winding number zero about the origin. In this case the third order asymptotics is

$$
\log \left|\operatorname{det} T_{n}(\varphi)\right|=n(\log |\varphi|)_{0}+A \log n+B+o(1) \quad \text { as } \quad n \rightarrow \infty,
$$

where $A$ and $B$ are completely identified constants [3].

\section{Normal operators}

In [15] it is shown that if $A \in \mathscr{F}\left(\left\{P_{n}\right\}\right)$ is selfadjoint, then (7) is true for $U=e^{A}$. Since in this case $U \in \mathscr{F}\left(\left\{P_{n}\right\}\right.$ and $\mathscr{H}(U)$ is some line segment $[m, M] \subset$ $(0, \infty)$, the result of [15] is a straightforward consequence of Corollary 3.1. Here is what Corollary 3.1 tells about normal operators. 
THEOREM 4.1. Let $A \in \mathscr{F}\left(\left\{P_{n}\right\}\right)$ be normal and put $U=e^{A}$. If the spectrum of $A$ is contained in some open horizontal strip of width $\pi$, that is, if there exists an $y_{0} \in \mathrm{R}$ such that $\left|\operatorname{Im} \lambda-y_{0}\right|<\pi / 2$ for all $\lambda \in \sigma(A)$, then the closure of $\mathscr{H}(U)$ does not contain the origin and

$$
\log \operatorname{det} P_{n} U P_{n}=\operatorname{tr} P_{n} A P_{n}+o\left(R_{n}\right) \quad \text { as } n \rightarrow \infty,
$$

where $\log$ is any branch of the logarithm that is analytic on clos $\mathscr{H}(U)$.

Proof. The operator $U$ is normal together with $A$, and $\sigma(U)$ is contained in some open half-plane whose boundary passes through the origin. The closure of the numerical range of the normal operator $U$ is the convex hull of $\sigma(U)$. Thus, 0 is not in clos $\mathscr{H}(U)$ and Corollary 3.1 gives (12).

EXAmple 4.2. This example shows that Theorem 4.1 is sharp. Let $A=$ $L(\psi)$ where $\psi(t)=i \pi / 2$ for $t$ on the upper half of the unit circle $\mathrm{T}$ and $\psi(t)=-i \pi / 2$ for $t$ on the lower half. Then $\sigma(A)=\{-i \pi / 2, i \pi / 2\}$. We have $U=e^{A}=L\left(e^{\psi}\right)$. Clearly, $\psi_{0}=0$. If (12) would be true, it would follow that $\log \left|\operatorname{det} T_{n}\left(e^{\psi}\right)\right|=o(n)$ or, equivalently, $\left|\operatorname{det} T_{n}\left(e^{\psi}\right)\right|^{1 / n} \rightarrow 1$ as $n \rightarrow \infty$. However, $T_{n}\left(e^{\psi}\right)$ is skew-symmetric (see, e.g., [6, p. 143]) and hence $\operatorname{det} T_{n}\left(e^{\psi}\right)=0$ whenever $n$ is odd.

EXAmPLE 4.3. This example reveals that Theorem 3.2 is in general no longer valid without the requirement that $\left\{P_{n} U^{k} P_{n}\right\}$ be stable. Let $U=L(\varphi)$ where $\varphi(t)=e^{i \pi / 4}$ for $t$ on the upper half of the unit circle $T$ and $\varphi(t)=e^{-i \pi / 4}$ for $t$ on the lower half. Then $\mathscr{H}(U)$ is the line segment between $e^{-i \pi / 4}$ and $e^{i \pi / 4}$. We have $U^{2}=L\left(\varphi^{2}\right)$ and $\varphi^{2}$ takes the values $i$ and $-i$ on the upper and lower halves of $\mathrm{T}$, respectively. This implies that $T_{n}\left(\varphi^{2}\right)$ is not stable. From Example 4.2 we know that $T_{n}\left(\varphi^{2}\right)$ is skew-symmetric and that therefore $\operatorname{det} T_{n}\left(\varphi^{2}\right)=0$ for odd $n$. Relation (8) amounts to

$$
\log \left|\operatorname{det} T_{n}\left(\varphi^{2}\right)\right|=2 \log \left|\operatorname{det} T_{n}(\varphi)\right|+o(n),
$$

and this is clearly not true because $\log \left|\operatorname{det} T_{n}(\varphi)\right|=n(\log |\varphi|)_{0}+o(n)$ due to Corollary 3.3 .

\section{Two more proofs for normal operators}

Here are two more proofs of Theorem 4.1.

SECOND PROOF. We proceed directly, without invoking Theorem 2.1. We know that the origin does not lie in the closure of $\mathscr{H}(U)$ and we may therefore without loss of generality assume that clos $\mathscr{H}(U)$ is contained in the right open half-plane. Let $\Gamma$ be a smooth curve in the right open half-plane that encircles 
clos $\mathscr{H}(U)$ exactly once counter-clockwise. Obviously, $\mathscr{H}\left(P_{n} U P_{n}\right)$ is a subset of $\mathscr{H}(U)$. Consequently,

$$
\begin{aligned}
& \frac{1}{R_{n}}\left(\log \operatorname{det} P_{n} U P_{n}-\operatorname{tr} P_{n} A P_{n}\right) \\
& =\frac{1}{R_{n}}\left(\operatorname{tr} \log P_{n} U P_{n}-\operatorname{tr} P_{n} A P_{n}\right) \\
& =\frac{1}{R_{n}}\left(\operatorname{tr} \log P_{n} e^{A} P_{n}-\operatorname{tr} \log e^{P_{n} A P_{n}}\right) \\
& =\frac{1}{2 \pi i R_{n}} \int_{\Gamma}(\log \lambda) \operatorname{tr}\left[\left(\lambda-P_{n} e^{A} P_{n}\right)^{-1}-\left(\lambda-e^{P_{n} A P_{n}}\right)^{-1}\right] d \lambda \\
& =\frac{1}{2 \pi i R_{n}} \int_{\Gamma}(\log \lambda) \operatorname{tr}\left[\left(\lambda-P_{n} e^{A} P_{n}\right)^{-1}\left(e^{P_{n} A P_{n}}-P_{n} e^{A} P_{n}\right)\right. \\
& \left.\quad \times\left(\lambda-e^{P_{n} A P_{n}}\right)^{-1}\right] d \lambda .
\end{aligned}
$$

Taking into account that $\left\|(\lambda-B)^{-1}\right\| \leq 1 / \operatorname{dist}(\lambda, \operatorname{clos} \mathscr{H}(B))$ for every operator $B \in \mathscr{B}(H)$, we get

$$
\begin{aligned}
\frac{1}{R_{n}} \mid \log \operatorname{det} P_{n} U & P_{n}-\operatorname{tr} P_{n} A P_{n} \mid \\
& \leq \frac{1}{2 \pi R_{n}}\left\|e^{P_{n} A P_{n}}-P_{n} e^{A} P_{n}\right\|_{1} \int_{\Gamma} \frac{|\log \lambda||d \lambda|}{\operatorname{dist}(\lambda, \operatorname{clos} \mathscr{H}(U))^{2}} .
\end{aligned}
$$

Finally, from (9) we obtain

$$
\begin{aligned}
\frac{1}{R_{n}}\left\|e^{P_{n} A P_{n}}-P_{n} e^{A} P_{n}\right\|_{1} & \leq\|A\| e^{\|A\|} \frac{\left\|P_{n} A Q_{n}\right\|_{1}}{R_{n}} \\
& \leq\|A\| e^{\|A\|} \frac{\left\|P_{n}\right\|_{2}\left\|P_{n} A Q_{n}\right\|_{2}}{R_{n}} \\
& =\|A\| e^{\|A\|} \frac{\sqrt{R_{n}}\left\|P_{n} A Q_{n}\right\|_{2}}{R_{n}},
\end{aligned}
$$

which goes to zero because $A \in \mathscr{F}\left(\left\{P_{n}\right\}\right)$.

ThIRD PROOF. We start with formula (19) of [15]. This formula is a generalization of Liouville's formula from ordinary differential equations and it says that

$$
\operatorname{det} P_{n} U P_{n}=e^{\operatorname{tr} P_{n} A P_{n}} \exp \left(\int_{0}^{1} \int_{0}^{t} E(t, \tau) d \tau d t\right)
$$


where

$$
E(t, \tau)=\operatorname{tr}\left(P_{n} A Q_{n} e^{(t-\tau) Q_{n} A Q_{n}} Q_{n} A P_{n} P_{n} e^{\tau A} P_{n}\left(P_{n} e^{t A} P_{n}\right)^{-1} P_{n}\right) .
$$

It follows that

$$
\begin{aligned}
|E(t, \tau)| & \leq e^{(t-\tau)\|A\|} e^{\tau\|A\|}\left\|\left(P_{n} e^{t A} P_{n}\right)^{-1} P_{n}\right\|\left\|P_{n} A Q_{n}\right\|_{2}\left\|Q_{n} A P_{n}\right\|_{2} \\
& =e^{t\|A\|}\left\|\left(P_{n} e^{t A} P_{n}\right)^{-1} P_{n}\right\|\left\|P_{n} A Q_{n}\right\|_{2}\left\|Q_{n} A P_{n}\right\|_{2} .
\end{aligned}
$$

Since $A \in \mathscr{F}\left(\left\{P_{n}\right\}\right)$, we obtain

(13) $\frac{1}{R_{n}}\left|\log \operatorname{det} P_{n} U P_{n}-\operatorname{tr} P_{n} A P_{n}\right|$

$$
\begin{aligned}
& \leq\left(\int_{0}^{1} t e^{t\|A\|}\left\|\left(P_{n} e^{t A} P_{n}\right)^{-1} P_{n}\right\| d t\right) \frac{\left\|P_{n} A Q_{n}\right\|_{2}}{\sqrt{R_{n}}} \frac{\left\|Q_{n} A P_{n}\right\|_{2}}{\sqrt{R_{n}}} \\
& =\left(\int_{0}^{1} t e^{t\|A\|}\left\|\left(P_{n} e^{t A} P_{n}\right)^{-1} P_{n}\right\| d t\right) o(1) .
\end{aligned}
$$

We write $A=B+i C$ with self-adjoint operators $B$ and $C$. Since $A$ is normal, the operators $B$ and $C$ commute, that is $B C=C B$. By assumption, $\sigma(A) \subset$ $[m, M] \times\left[y_{0}-h, y_{0}+h\right]$ for certain $-\infty<m<M<\infty$ and $0<h<\pi / 2$. This implies that $\sigma(B) \subset[m, M]$ and $\sigma(C) \subset\left[y_{0}-h, y_{0}+h\right]$. Therefore

$$
\begin{aligned}
\left|\left(P_{n} x, e^{t A} P_{n} x\right)\right| & =\left|\left(e^{\frac{t}{2} B} P_{n} x, e^{i t C} e^{\frac{t}{2} B} P_{n} x\right)\right| \\
& \geq \cos (t h)\left|\left(P_{n} x, e^{t B} P_{n} x\right)\right| \\
& \geq \cos (t h) e^{t m}\left\|P_{n} x\right\|^{2}
\end{aligned}
$$

for all $n$ and thus,

$$
\left\|\left(P_{n} e^{t A} P_{n}\right)^{-1} P_{n}\right\| \leq \frac{1}{e^{t m} \cos t h} .
$$

But if $0 \leq t \leq 1$, then

$$
\frac{1}{e^{t m} \cos t h} \leq \frac{1}{e^{-|m|} \cos h} .
$$

Inserting (15), (16) in (13) we arrive at the assertion.

If in the foregoing proof we just wanted $\left(P_{n} e^{t A} P_{n}\right)^{-1}$ to exist we could allow $m=-\infty$. To see this, assume there is some nonzero $x \in \operatorname{ran} P_{n}$ such that $P_{n} e^{t A} P_{n} x=0$. Estimating as in (14) we get

$$
0=\left(P_{n} x, e^{t B} P_{n} x\right)=\left\|e^{\frac{t}{2} B} P_{n} x\right\|^{2},
$$


which shows that $e^{\frac{t}{2} B} P_{n} x=0$. Repeating this argument we arrive at

$$
0=\left(P_{n} x, e^{\frac{t}{2} B} P_{n} x\right)=\left\|e^{\frac{t}{4} B} P_{n} x\right\|^{2}
$$

and hence $e^{\frac{t}{4} B} P_{n} x=0$. Proceeding further in this way and using the strong continuity of $e^{t B}$ we eventually obtain that $x=0$. Although in this case of semi-bounded $B$ the invertibility of $P_{n} e^{t A} P_{n}$ is still ensured, the uniform bound, which is needed for stability, is not necessarily valid.

With a view to Example 4.2 it is not surprising that the assumption on $C$ cannot be weakened in general. For instance, in the special case $B=0$, where $A=i C$ and hence $e^{t A}$ is unitary, a gap condition has to be imposed on $\sigma(A)$ : when $\sup \sigma\left(P_{n} C P_{n}\right)<\inf \sigma\left(Q_{n} C Q_{n}\right)$ or $\sup \sigma\left(Q_{n} C Q_{n}\right)<\inf \sigma\left(P_{n} C P_{n}\right)$, then $\left(P_{n} e^{t A} P_{n}\right)^{-1}$ exists for all $t \in \mathrm{R}$ whereas one can construct counterexamples in the case where such a gap is missing (see [16, Theorem 3.4, Corollary 3.6, and Section 5]).

\section{REFERENCES}

1. Arveson, W., The role of $C^{*}$-algebras in infinite-dimensional numerical linear algebra, Contemp. Math. 167 (1994), 114-129.

2. Arveson, W., $C^{*}$-algebras and numerical linear algebra, J. Funct. Anal. 122 (1994), 333-360.

3. Basor, E. L., A localization theorem for Toeplitz determinants, Indiana Univ. Math. J. 28 (1979), 975-983.

4. Bédos, E., On Folner nets, Szegó's theorem and other eigenvalue distribution theorems, Exposition. Math. 15 (1997), 193-228.

5. Böttcher, A., and Silbermann, B., Analysis of Toeplitz Operators, Akademie-Verlag, Berlin 1989 and Springer-Verlag, Berlin 1990.

6. Böttcher, A., and Silbermann, B., Introduction to Large Truncated Toeplitz Matrices, SpringerVerlag, New York 1999.

7. Brown, N. P., Quasidiagonality and the finite section method, Preprint math. NA/0312316.

8. Dym, H., Trace formulas for a class of Toeplitz-like operators, Israel J. Math. 27 (1977), $21-48$.

9. Dym H., and Ta'assan, S., An abstract version of a limit theorem of Szegö, J. Funct. Anal. 43 (1981), 294-312.

10. Gohberg, I., and Feldman, I. A., Convolution Equations and Projection Methods for Their Solution, Amer. Math. Soc., Providence, R.I., 1974.

11. Guillemin, V., Some classical theorems in spectral theory revisited, In: Seminar on Singularities of Solutions of Linear Partial Differential Equations, 219-259, Princeton Univ. Press 1979.

12. Hagen, R., Roch, S., and Silbermann, B., $C^{*}$-Algebras and Numerical Analysis, Marcel Dekker, New York 2001.

13. Krein, M. G., and Spitkovsky, I. M., Some generalizations of Szegö's first limit theorem, Anal. Math. 9 (1983), 23-41 [Russian].

14. Laptev, A., and Safarov, Yu., Szegó type limit theorems, J. Funct. Anal. 138 (1996), 544-559.

15. Otte, P., An abstract Szegó theorem, J. Math. Anal. Appl. 289 (2004), 167-179.

16. Otte, P., Section determinants of unitary operators and the operator-valued Riccati differential equation, Math. Proc. Cambridge Philos. Soc. 133 (2002), 373-382. 
17. SeLegue, D., A $C^{*}$-algebraic extension of the Szegö trace formula, Talk given at the GPOTS, Arizona State University, Tempe, May 22, 1996.

18. Szegő, G., Ein Grenzwertsatz über die Toeplitzschen Determinanten einer reellen positiven Funktion, Math. Ann. 76 (1915), 490-503.

19. Widom, H., Asymptotic behavior of block Toeplitz, matrices and determinants, II, Adv. Math. 21 (1976), 1-29.

20. Widom, H., Asymptotic expansions of determinants for families of trace class operators, Indiana Univ. Math. J. 27 (1978), 449-478.

21. Widom, H., Szegô's limit theorem: the higher-dimensional matrix case, J. Funct. Anal. 39 (1980), 182-198.

FAKULTÄT FÜR MATHEMATIK

TU CHEMNITZ

D-09107 CHEMNITZ

GERMANY

E-mail: aboettch@mathematik.tu-chemnitz.de
FAKULTÄT FÜR MATHEMATIK RUHR-UNIVERSITÄT BOCHUM UNIVERSITÄTSSTR. 150 D-44801 BOCHUM GERMANY

E-mail: peter.otte@ruhr-uni-bochum.de 